\title{
Resultados funcionales a largo plazo de la cirugía abierta de liberación del túnel carpiano
}

\author{
Guillermo Rojas Duque, ' Christian J. González Bateca, ' Alejandro Torres Mayorga, ' Rómulo A. Hernández Ramírez, \\ Sofía E. Muñoz Medina* \\ "Servicio de Ortopedia y Traumatología, Fundación Universitaria Sanitas, Clínica Universitaria Colombia, Bogotá, Colombia \\ **Unidad de Investigaciones, Fundación Universitaria Sanitas, Bogotá, Colombia
}

\begin{abstract}
RESUMEN
Introducción: El síndrome del túnel carpiano es la neuropatía por atrapamiento más común que genera compresión del nervio mediano. La cirugía de liberación abierta del nervio mediano tiene un papel importante, especialmente, en pacientes que no responden al manejo conservador o con diagnóstico de síndrome del túnel carpiano con criterios de gravedad. El propósito de este estudio fue describir los resultados funcionales, la satisfacción y la fuerza objetiva a mediano (6-24 meses) y largo plazo (>24 meses) con la técnica abierta convencional en la población local. Materiales y Métodos: Estudio observacional descriptivo con datos retrospectivos de resultados clínicos funcionales a mediano y largo plazo en pacientes sometidos a cirugía de liberación abierta del nervio mediano como tratamiento del síndrome del túnel carpiano. Se determinaron el nivel funcional según el BCTQ y la FSS, la fuerza de agarre con un dinamómetro electrónico y la satisfacción. Resultados: Se realizaron 100 procedimientos entre mayo de 2012 y septiembre de 2018, con un seguimiento posoperatorio $>6$ meses. La mayoría eran mujeres (83\%) con una mediana de la edad de 59 años. El $97 \%$ obtuvo resultados buenos y excelentes a mediano plazo y el $90 \%$, a largo plazo, con una mediana de fuerza de $17 \mathrm{~kg}$ (RIC 7,4) y una satisfacción de 90 (RIC 20) a mediano y largo plazo. Conclusiones: La cirugía abierta de liberación del nervio mediano en pacientes con síndrome del túnel carpiano logra resultados buenos y excelentes a mediano y largo plazo en cuanto a funcionalidad, fuerza y satisfacción.
\end{abstract}

Palabras clave: Síndrome del túnel carpiano; electromiografía; fuerza muscular; fuerza de la mano, satisfacción.

Nivel de Evidencia: IV

\section{Long-term Functional Outcomes of Open Carpal Tunnel Release Surgery}

\section{ABSTRACT}

Introduction: Carpal tunnel syndrome (CTS) is the most common entrapment neuropathy that causes compression of the median nerve. Open median nerve release surgery plays an important role, especially in patients with failed conservative management or with a diagnosis of severe CTS. The purpose of the following study is to describe the functional outcomes, satisfaction, and objective strength in the medium ( 6 to 24 months) and long term (greater than 24 months) with the conventional open technique in the local population. Materials and Methods: Descriptive observational study based on retrospective data of functional clinical outcomes in the medium and long term in patients undergoing open release surgery of the median nerve as a treatment for CTS. Functional level according to the BCTQ and FSS, grip strength with an electronic dynamometer, and satisfaction were determined. Results: 100 procedures were performed between May 2012 and September 2018, with a follow-up of more than 6 months. The majority were women (83\%) with a total median age of 59 years showing good to excellent results in the $97 \%$ in the medium term and $90 \%$ in the long term, with a median strength of $17 \mathrm{~kg}$ (Interquartile range: 7.4 ) and satisfaction of 90 (Interquartile range: 20). Conclusions: Open surgery to release the median nerve in patients with CTS shows good to excellent functional outcomes, satisfaction, and strength in the medium and long term.

Keywords: Carpal tunnel syndrome; electromyography; muscle strength dynamometer; hand strength; operative surgical procedures; treatment result.

Level of Evidence: IV

\footnotetext{
Recibido el 25-1-2021. Aceptado luego de la evaluación el 5-6-2021 • Dr. GUILLERMO ROJAS DUQUE • grojasduque @ hotmail.com Cómo citar este artículo: Rojas Duque G, González Bateca CJ, Torres Mayorga A, Hernández Ramírez RA, Muñoz Medina SE. Resultados funcionales a largo plazo de la cirugía abierta de liberación del túnel carpiano. Rev Asoc Argent Ortop Traumatol 2021;86(5):601-612. https://doi.org/10.15417/issn. 1852-7434.2021.86.5.1305 


\section{INTRODUCCIÓN}

El síndrome del túnel carpiano (STC) es la neuropatía por atrapamiento más común que genera compresión y tracción del nervio mediano. Se estima una prevalencia del 4-5\% en la población general, con un pico de incidencia entre los 40 y 60 años, y un predominio en las mujeres. ${ }^{1}$ El cuadro puede ser unilateral o bilateral y provoca síntomas, como parestesias, ardor, pesadez o dolor en el territorio inervado por el nervio mediano, que pueden irradiarse al antebrazo, al codo e incluso al hombro; síntomas de predominio nocturno o matutino, o desencadenados por posiciones prolongadas y de carácter progresivo. ${ }^{2,3}$ Su etiología se considera multifactorial, tal vez los factores anatómico-estructurales y genéticos son más importantes que los factores ocupacionales o de uso repetitivo. ${ }^{4,5}$

El diagnóstico del STC se basa en hallazgos semiológicos, según las características clínicas de los síntomas y signos. La prueba de Tinel tiene una sensibilidad del $26-79 \%$ y una especificidad del $40-100 \% .{ }^{6}$ La prueba de Phalen que busca generar parestesias con la flexión de la muñeca tiene una sensibilidad del 46-80\% y una especificidad del 51-91\%. ${ }^{7}$ Asimismo, se ha descrito la prueba de Durkan con la cual se reproducen los síntomas mediante la compresión del carpo, y que es más sensible y específica que las pruebas anteriores. ${ }^{8}$ Las parestesias nocturnas tienen una sensibilidad del $96 \% .^{9}$ A falta de un patrón de referencia para el diagnóstico, se deben utilizar todas las medidas de diagnóstico razonables, incluidos los signos, los síntomas, la electromiografía y la neuroconducción, que incrementarán la precisión del diagnóstico y además permitirán estratificar la gravedad del STC para generar estrategias de tratamiento. ${ }^{10}$

Entre las múltiples opciones terapéuticas para abordar este cuadro, se pueden mencionar la prevención, las férulas, la terapia física, las infiltraciones, las modificaciones de la actividad o la cirugía abierta o endoscópica. ${ }^{1,3}$ El manejo quirúrgico consiste en la liberación del contenido del túnel carpiano con un corte longitudinal del ligamento transverso del carpo reduciendo la presión dentro del túnel mediante una técnica abierta (incisión longitudinal larga de la muñeca y visualización directa del ligamento), “mini-open” (incisión corta) o técnica endoscópica. ${ }^{11}$ No se han demostrado diferencias significativas en los resultados funcionales a largo plazo comparando la técnica abierta y la endoscópica. ${ }^{12-14}$

La técnica abierta empleada y el uso de cuestionarios o escalas aplicados en el posoperatorio resultan útiles, pues demuestran que la técnica abierta sigue vigente y, en segundo lugar, permiten cuantificar, objetivar y seguir los resultados en estudio. Por esta razón, pese a que hay múltiples ensayos publicados con diferentes períodos de seguimiento y métodos quirúrgicos, en este estudio, se resalta la eficacia de la técnica abierta convencional y se describe si los resultados en la población local son similares a los descritos previamente.

En investigación clínica, la definición de seguimiento es muy variable y depende del tipo de enfermedad, el tratamiento y la población en estudio, los textos tienden a polarizar corto o largo plazo; según la fuente citada, se consideran dichos intervalos de tiempo en meses o años indistintamente. Domínguez y cols. consideran como corto plazo a un período de un mes, ${ }^{15}$ Ishida y cols. contemplan un mediano plazo de los tres meses a un período de cinco años ${ }^{16}$ y largo plazo desde los dos años hasta incluso nueve años, según Louie y cols., Kouyoumdjian y cols., y Tang y cols., ${ }^{17-19}$ respectivamente. Dada la variabilidad en los tiempos de estas definiciones, se tomaron como referencia dos estudios: corto plazo ( $<6$ meses) y mediano plazo (de 6 a 24 meses), según Domínguez y cols., ${ }^{15}$ y largo plazo (>24 meses), según Louie y cols. ${ }^{17}$

El propósito de este estudio fue describir los resultados funcionales, la satisfacción y la fuerza objetiva a mediano (6-24 meses) y largo plazo (>24 meses) con la técnica abierta convencional de liberación del nervio mediano por STC en la población local, efectuada por un cirujano de mano en una clínica universitaria.

\section{MATERIALES Y MÉTODOS}

Se llevó a cabo un estudio observacional descriptivo a partir de datos retrospectivos de pacientes operados en una clínica universitaria, teniendo en cuenta unos períodos de tiempo estipulados aleatoriamente por el grupo investigador y en función de la duración del estudio dada la heterogeneidad de los lapsos, definidos como mediano plazo (6-24 meses) y largo plazo (25-70 meses), según los estudios de Domínguez y cols., y Louie y cols. ${ }^{15,17}$

Los criterios de inclusión fueron: pacientes $\geq 18$ años sometidos a una cirugía primaria de liberación abierta del túnel carpiano operados por un único ortopedista cirujano de mano. Se excluyó a aquellos con cirugía de revisión 
de liberación del nervio mediano, STC agudo por trauma o infección, secundario a masas, técnica endoscópica y neuropatía alta del nervio mediano concomitante y aquellos que se perdieron en el seguimiento por no contar con la evaluación mediante el Boston Carpal Tunnel Syndrome Questionnaire (BCTQ), la Functional Status Scale (FSS) y de satisfacción global o que no realizaron la prueba de fuerza de agarre con un dinamómetro en algún momento del control en un tiempo $>6$ meses del posoperatorio.

El estudio fue aprobado por el comité de ética institucional. La evaluación clínica y la recolección de los datos estuvieron a cargo del equipo investigador (cirujano tratante y dos residentes de ortopedia de tercer año). Los pacientes fueron incluidos, de manera retrospectiva, desde mayo de 2012 hasta septiembre de 2018, el seguimiento mínimo fue de 6 meses. Se revisaron las historias clínicas para verificar que el paciente hubiera realizado las evaluaciones con el BCTQ, la FSS y de satisfacción global expresada en porcentaje, donde se le preguntaba: si tuviera que tomar la decisión de operarse, ¿lo haría de nuevo? Con un sí o un no como respuesta. Finalmente se realizó una prueba de fuerza de agarre con un dinamómetro portátil (CAMRY EH101) registrando los resultados en $\mathrm{kg}$ de fuerza de ambas manos sin tener en cuenta la dominancia.

El BCTQ consta de 11 preguntas que evalúan las características clínicas y la gravedad del STC, está validado al español ${ }^{20}$ y se utilizó como medio para determinar la mejoría de los síntomas en pacientes ya diagnosticados y operados. ${ }^{21,22}$ Ligada al BCTQ está la FSS que evalúa el estado funcional según las actividades cotidianas. ${ }^{23}$

La satisfacción de los pacientes se comunica del $0 \%$ al 100\% calculando la media y el porcentaje de pacientes que volverían a operarse teniendo en cuenta la mejoría de sus síntomas; la fuerza se informa en kg de fuerza de agarre.

\section{Análisis estadístico}

Los resultados del BCTQ, la FSS, los porcentajes de satisfacción y agarre medido en kg se analizaron de manera descriptiva, y se comunican como frecuencias absolutas y relativas a las variables cualitativas. Las variables cuantitativas se presentan a través de medidas de tendencia central y dispersión según la distribución de los datos por medio de la prueba de Shapiro-Wilk. Se efectuó un análisis exploratorio de comparación de mediano y largo plazo donde las variables cualitativas se compararon con la prueba de ji al cuadrado y las variables cuantitativas, con la prueba U de Mann-Whitney, dada la distribución de los datos, ninguna tenía una distribución normal. Se consideró estadísticamente significativo un valor $\mathrm{p}<0,05$. Los datos se analizaron con el programa estadístico Stata 15 .

\section{Técnica quirúrgica}

La técnica quirúrgica empleada consiste en un abordaje abierto, siguiendo un orden sistemático y usando como referencia las líneas de Kaplan. ${ }^{1}$ Se ubica al paciente en decúbito supino con el hombro en abducción y el miembro superior apoyado en la mesa de mano. Se coloca un torniquete neumático elevado a un promedio de 250 mmHg en función de la presión arterial sistólica. El procedimiento se realiza bajo anestesia general, sedación o anestesia local. Se infiltra la zona quirúrgica con lidocaína más bupivacaína sin epinefrina $4 \mathrm{cc} \mathrm{de} \mathrm{cada} \mathrm{una,} \mathrm{en}$ dos puntos, uno proximal llegando entre el flexor radial del carpo y el palmar largo al espacio de Parona a unos $2 \mathrm{~cm}$ del pliegue distal de la muñeca, se realiza de proximal a distal con una inclinación de $45^{\circ}$, y otro punto de infiltración distal en el extremo proximal de la incisión generando un habón en la zona quirúrgica (Figura 1), incisión a nivel del túnel del carpo de 2 a $3 \mathrm{~cm}$ según las referencias de Kaplan (Figura 2), disección de la piel, tejido celular subcutáneo hasta llegar al retináculo transverso del carpo (Figura 3). Se incide con una hoja de bisturí con el corte hacia arriba protegiendo el nervio hasta la grasa distal evitando lesionar el arco arterial (Figura 4). Hacia proximal se introduce una sonda acanalada entre el nervio mediano y el retináculo residual y, con la hoja del bisturí, se incide el retináculo usando la sonda como riel (Figura 5). Se corrobora la liberación completa con maniobra digital y se libera el nervio del borde radial y cubital del retináculo, se retira el torniquete y se realiza hemostasia antes del cierre (Figura 6). Se coloca una férula palmar corta que permite la movilidad completa de las articulaciones metacarpofalángicas (Figura 7) que se mantiene por ocho días al igual que los puntos. La cicatriz quirúrgica es pequeña y estéticamente adecuada (Figura 8). 


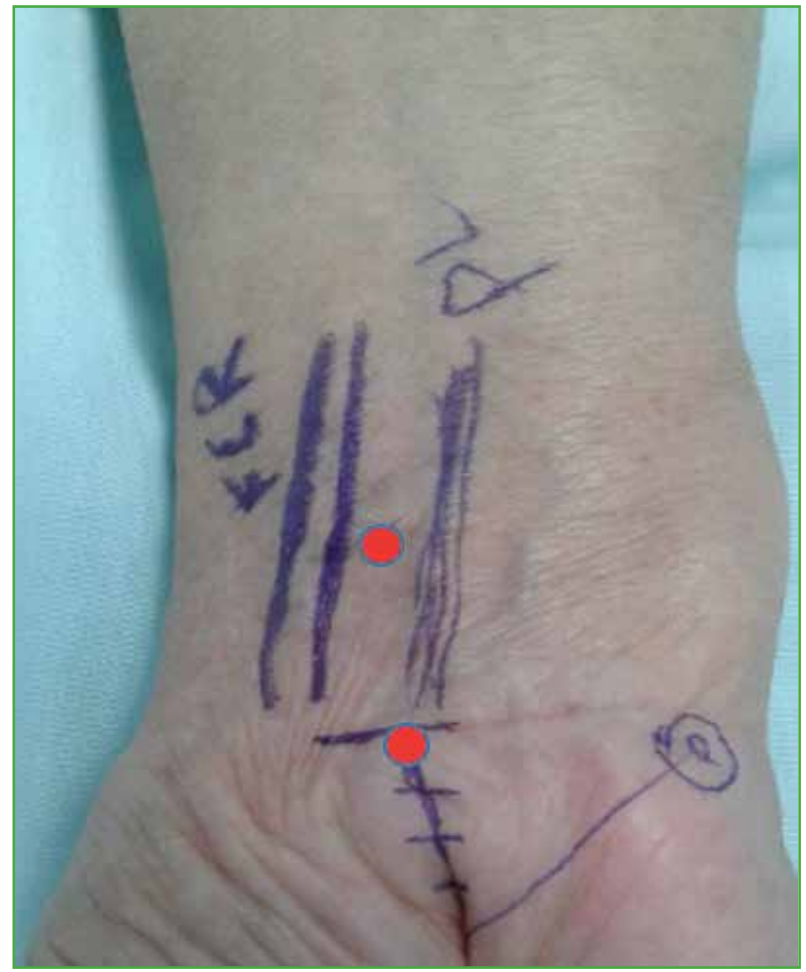

Figura 1. Infiltración de la zona quirúrgica. Los puntos rojos corresponden a la zona de infiltración. $\mathrm{PL}=$ palmaris longus, $\mathrm{FCR}=$ flexor carpi radialis, $\mathrm{P}=$ pisiforme.

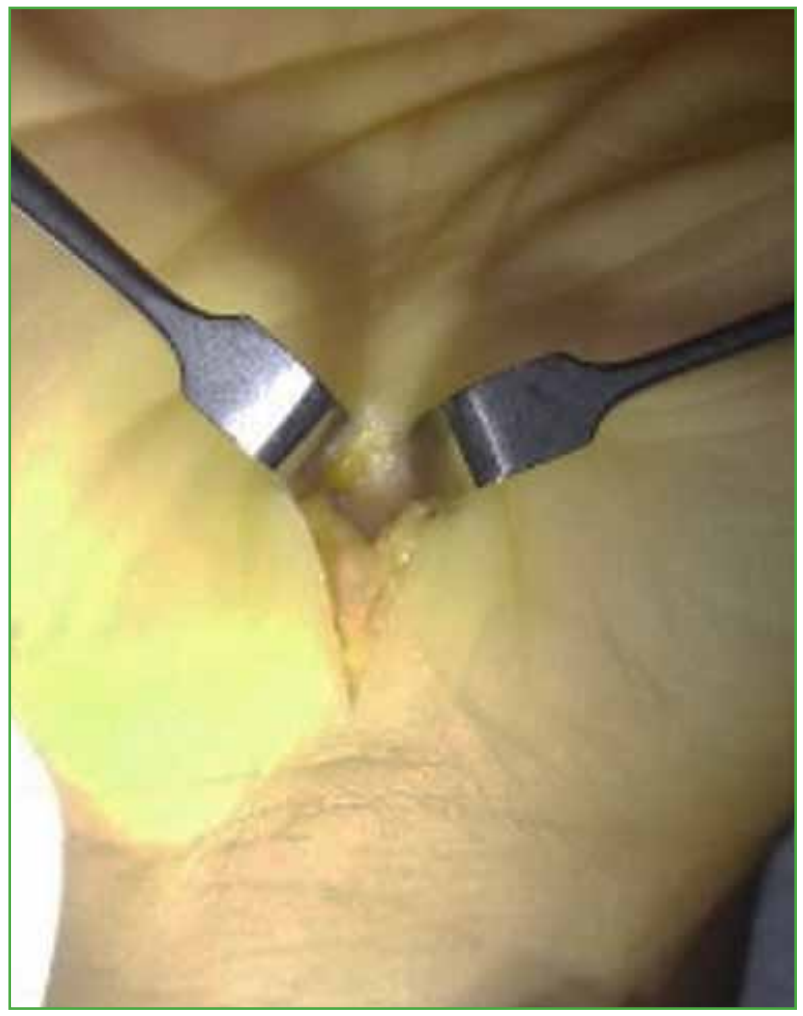

Figura 3. Disección superficial.

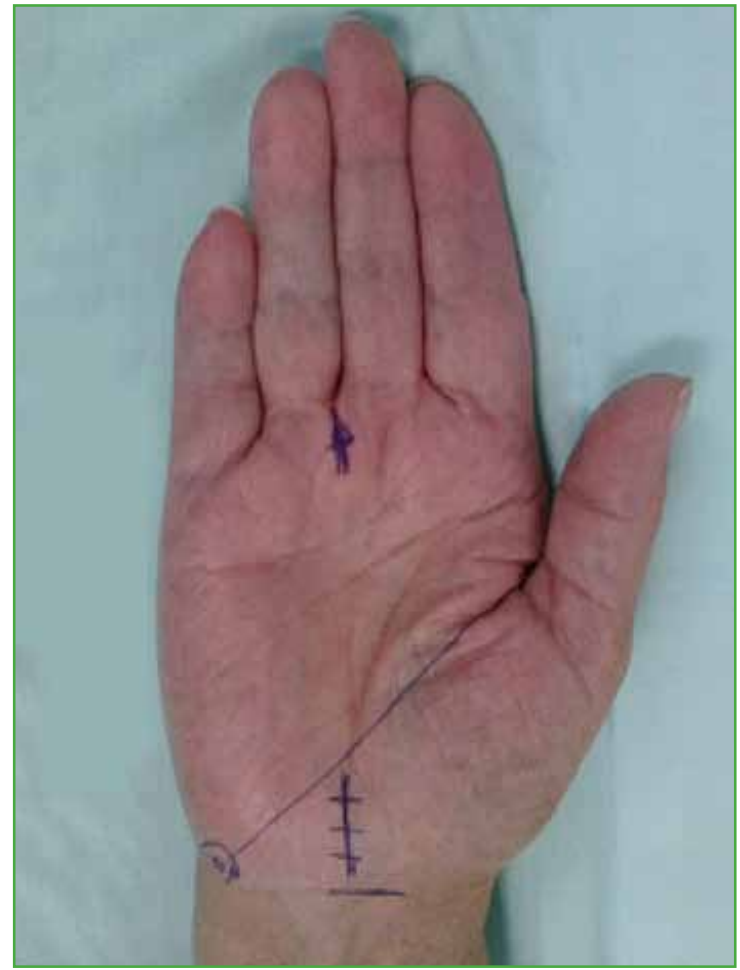

Figura 2. Abordaje según las líneas de Kaplan.

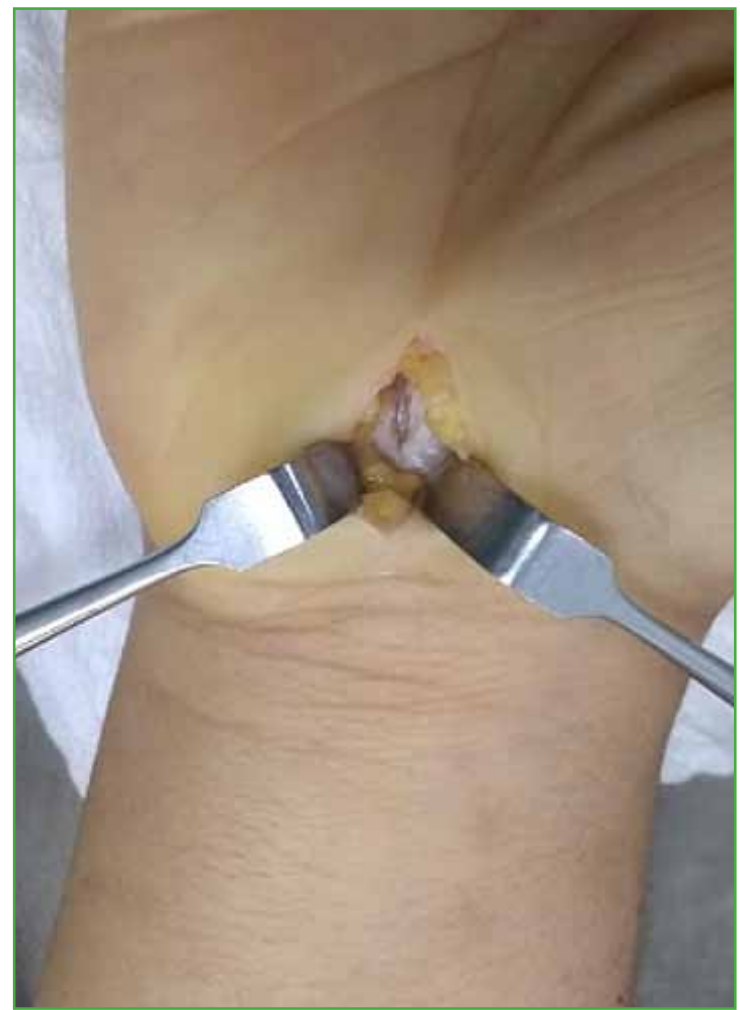

Figura 4. Disección profunda. 


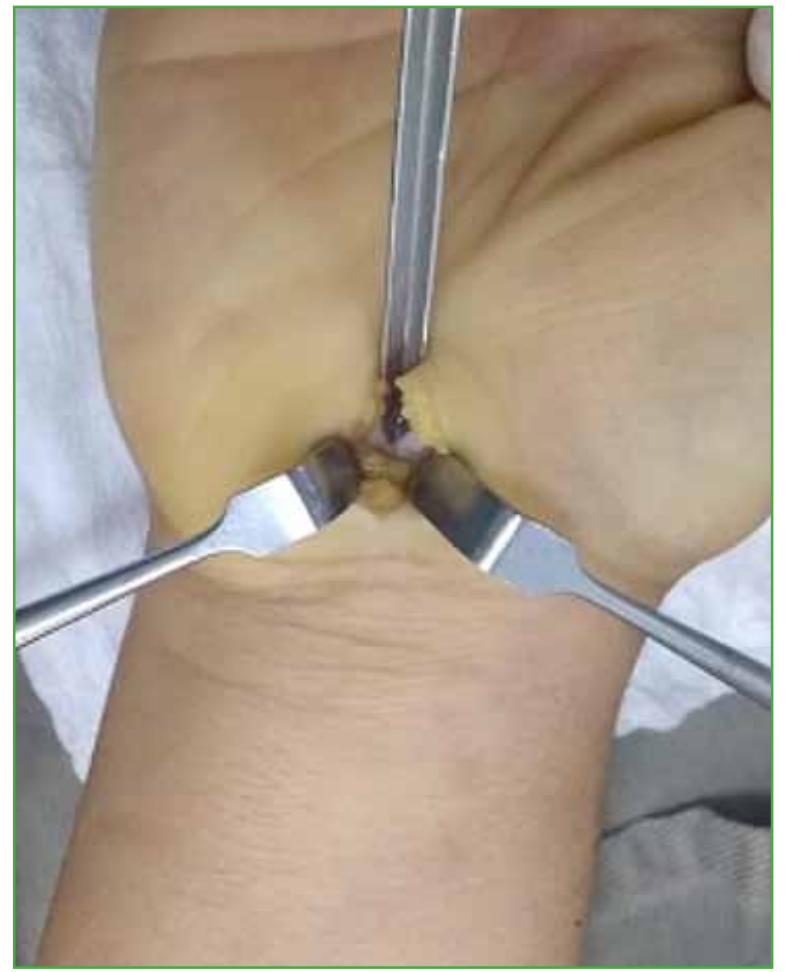

Figura 5. Introducción de la sonda acanalada entre el nervio mediano y el retináculo residual.
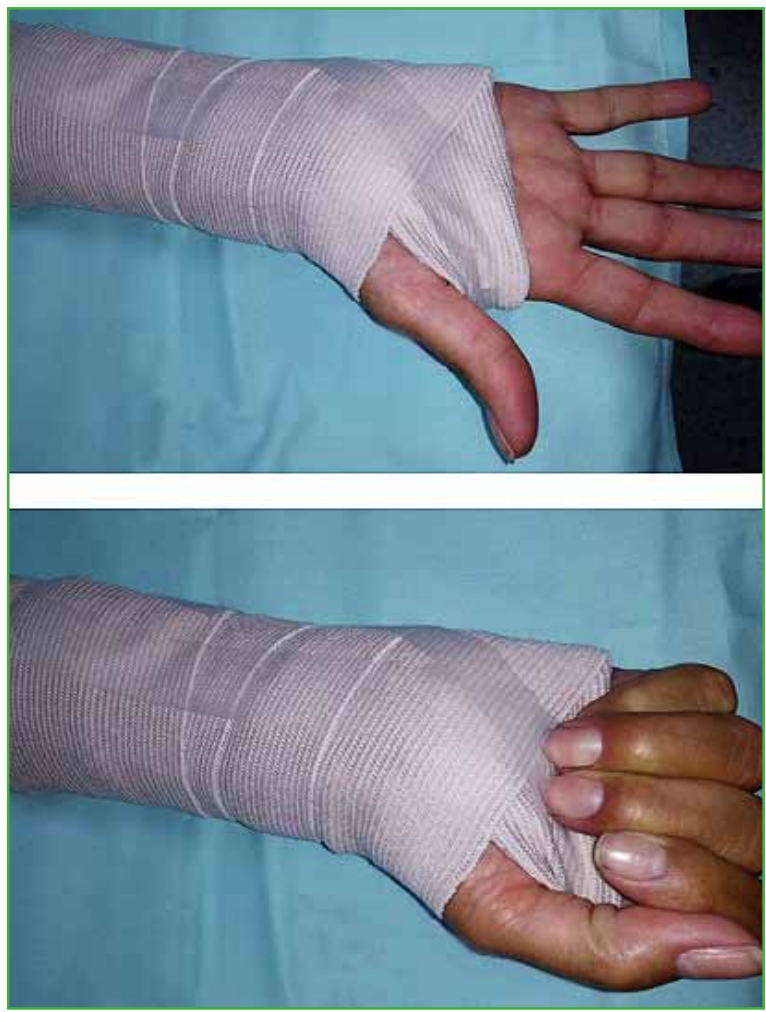

Figura 7. Inmovilización con férula palmar corta que permite la movilidad completa de las articulaciones metacarpofalángicas.

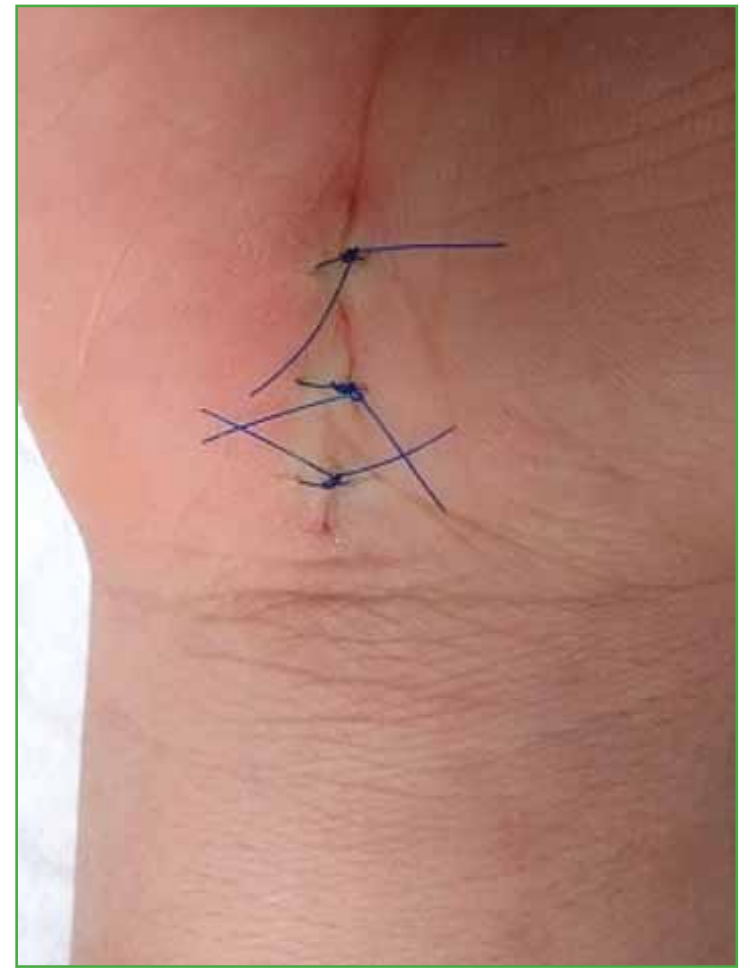

Figura 6. Cierre del procedimiento quirúrgico.

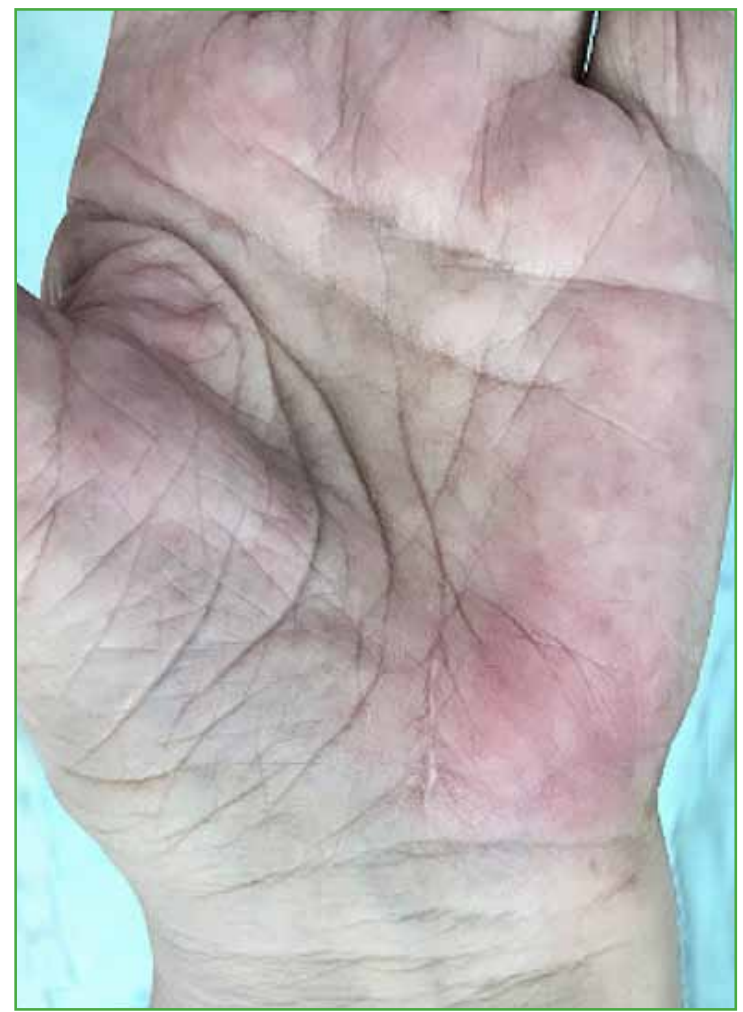

Figura 8. Cicatriz pequeña y estéticamente adecuada. 


\section{RESULTADOS}

Se recolectó información de 100 procedimientos de cirugía abierta de liberación del nervio mediano llevados a cabo en 81 pacientes que cumplieron con los criterios de inclusión y de no exclusión. Se excluyó a 78 pacientes, porque no contaban con los resultados del BCTQ, la FSS, de satisfacción global y ni con la prueba de agarre con un dinamómetro. Los pacientes incluidos eran 14 hombres $(17,2 \%)$ y 67 mujeres $(82,8 \%)$. En el $97 \%$ de los procedimientos, se midió el agarre objetivo con el dinamómetro. El 3\% restante de los pacientes dio su asentimiento por vía telefónica y se obtuvo información del BCTQ y la FSS. La edad promedio era de 60 años (rango 31-88). El $62 \%$ de los procedimientos fueron en la mano derecha y el $38 \%$, en la mano izquierda. En los 100 procedimientos, se utilizaron diferentes técnicas anestésicas: anestesia local controlada como está descrita en la técnica quirúrgica (86\%), anestesia local (7\%) y bloqueo venoso (7\%). La anestesia local resultó segura, proporcionó un adecuado efecto anestésico durante el procedimiento quirúrgico y un buen control del dolor en el posoperatorio. Por lo anterior, la técnica anestésica utilizada hace posible que la liberación del nervio mediano con la técnica abierta se pueda realizar en una sala de procedimientos y no necesariamente en un quirófano, lo que disminuye los costos y el tiempo quirúrgico.

La mediana de seguimiento fue de 38 meses (rango intercuartílico [RIC] 24), la mediana del puntaje en la escala de Boston fue 15 (RIC 11): bueno (25 pacientes) y excelente (56 pacientes); la mediana de la escala funcional fue 11 (RIC 6), en la mayoría de los casos, fue excelente (77\%) y, en el 15\%, bueno. La mediana de la fuerza de agarre de ambas extremidades cuantificada con un dinamómetro electrónico de mano fue de $17 \mathrm{~kg}(\mathrm{RIC} 7,4)$ y la mediana de la fuerza contralateral en las manos no operadas (medida solo en 57 pacientes), de $18 \mathrm{~kg}$ (RIC 5,3). Se logró recolectar información de los resultados electromiográficos antes de la cirugía en el 63\%, el 75\% de ellos correspondía a enfermedad grave y el $25 \%$, a enfermedad moderada, no había casos operados con enfermedad leve. La evaluación de la satisfacción arrojó una mediana de 90 (RIC 20), ningún paciente refirió $0 \%$ de mejoría, de los 17 pacientes con resultados malos, 15 se volverían a someter a la cirugía. El 94\% se volvería a operar teniendo en cuenta la mejoría de sus síntomas (Tabla 1).

\section{Resultados de diferencias y similitudes entre la gravedad}

La mayoría de la muestra estaba constituida por mujeres, lo que evidenció que, a mayor edad, los síntomas eran más severos. El grupo con síntomas moderados tuvo una mediana de seguimiento de 36 meses (RIC 36), una mediana en la escala de Boston de 12 (RIC 11), un puntaje de satisfacción de 90 (RIC 20), una mediana de fuerza en el lado afectado de $18,4 \mathrm{~kg}$ (RIC 5,2) y una mediana de fuerza contralateral de $18,3 \mathrm{~kg}$ (RIC 8,4), mientras que el grupo con síntomas severos tuvo una mediana de seguimiento de 38 meses (RIC 24), una mediana en la escala de Boston de 14 (RIC 8), un puntaje de satisfacción de 95 (RIC 20), una mediana de fuerza en el lado afectado de $18,2 \mathrm{~kg}(\mathrm{RIC} 9,4)$ y una mediana de fuerza contralateral de 18,2 $\mathrm{kg}$ (RIC 9,4). Los desenlaces en pacientes con síntomas moderados y graves fueron similares (Tabla 2).

\section{Resultados según el tiempo de seguimiento}

Los pacientes con un seguimiento a mediano plazo tenían una mediana de la edad de 64 años (RIC 18), con una mediana de seguimiento de 12.5 meses (RIC 16), una mediana en la escala de Boston de 13 (RIC 8); los resultados fueron excelentes en el $73 \%$ y buenos en el 15\%, el puntaje de la escala funcional fue 10 (RIC 3), resultado excelente en el 79\% de este grupo. El puntaje de satisfacción fue 90 (RIC 20), la mediana de la fuerza fue de 16,7 $\mathrm{kg}($ RIC 8,4) y la fuerza contralateral, $16,5 \mathrm{~kg}$ (RIC 6,1) similar en las dos extremidades. En el grupo con seguimiento a largo plazo, la mediana de la edad fue de 58 años (RIC 10), con una mediana de seguimiento a largo plazo de 48 meses (RIC 20), una mediana en la escala de Boston de 16 (RIC 11), los resultados fueron excelentes en el 47\%, cifra inferior a la del grupo de mediano plazo, y buenos en el 33\%; el puntaje de la escala funcional fue 11 (RIC 7), resultado excelente en el 76\%, porcentaje similar al del grupo de mediano plazo; la mediana de fuerza fue de $18 \mathrm{~kg}$ (RIC 8,3) y la fuerza contralateral, de $19 \mathrm{~kg}$ (RIC 5,1) (Tabla 3). 
Tabla 1. Descripción de las variables

Variable

Edad en años, mediana (RIC)

Sexo
Masculino
Femenino
$\begin{aligned} & \text { Lateralidad } \\ & \text { Derecha } \\ & \text { Izquierda }\end{aligned}$
Clasificación de la gravedad
Moderada
Severa

\section{Electromiografía}

Sí

Anestesia
Local
Bloqueo venoso
General

Tiempo de seguimiento en meses, mediana (RIC)

Escala de Boston, mediana (RIC)

Clasificación en la escala de Boston

Malo

Bueno

Excelente

Escala funcional

Clasificación en la escala funcional

Malo

Regular

Bueno

Excelente

Satisfacción, mediana (RIC)

Fuerza, mediana (RIC)

Fuerza contralateral $(\mathrm{n}=57)$, mediana $(\mathrm{RIC}) \quad 18(5,3)$

Se volvería a operar

Sí

No
17 (17)

27 (27)

$56(56)$

$11(6)$

$17(17)$

$83(83)$

$62(62)$

38 (38)

15 (15)

48 (48)

63 (63)

86 (86)

7 (7)

7 (7)

38 (24)

15 (11)

4 (4)

15 (15)

77 (77)

90 (20)

$17(7,4)$

94 (94)

6 (6)

Datos numéricos reportados con mediana y rango intercuartílico (RIC), dado un resultado de la prueba de Shapiro-Wilk $\mathrm{p}<0,05$. 
Tabla 2. Descripción de las variables según la gravedad

\begin{tabular}{|c|c|c|}
\hline Variable & Moderada (\%) & Severa $(\%)$ \\
\hline Edad en años, mediana (RIC) & $53(15)$ & $59(13)$ \\
\hline $\begin{array}{l}\text { Sexo } \\
\text { Masculino } \\
\text { Femenino }\end{array}$ & $\begin{array}{c}2(13) \\
13(87)\end{array}$ & $\begin{array}{c}7(15) \\
41(85)\end{array}$ \\
\hline $\begin{array}{l}\text { Lateralidad } \\
\text { Derecha } \\
\text { Izquierda }\end{array}$ & $\begin{array}{c}11(73) \\
4(27)\end{array}$ & $\begin{array}{l}32(67) \\
16(33)\end{array}$ \\
\hline $\begin{array}{l}\text { Anestesia } \\
\text { Local } \\
\text { Bloqueo venoso } \\
\text { General }\end{array}$ & $\begin{array}{l}11(73) \\
2(13) \\
2(13)\end{array}$ & $\begin{array}{c}40(83) \\
4(8) \\
4(8)\end{array}$ \\
\hline Tiempo de seguimiento en meses, mediana (RIC) & $36(36)$ & $38(24)$ \\
\hline Escala de Boston, mediana (RIC) & $12(11)$ & $14(8)$ \\
\hline $\begin{array}{l}\text { Clasificación en la escala de Boston } \\
\text { Malo } \\
\text { Bueno } \\
\text { Excelente }\end{array}$ & $\begin{array}{c}2(13) \\
3(20) \\
10(67)\end{array}$ & $\begin{array}{l}7(15) \\
12(25) \\
29(60)\end{array}$ \\
\hline Escala funcional & $10(5)$ & $10(6)$ \\
\hline $\begin{array}{l}\text { Clasificación en la escala funcional } \\
\text { Malo } \\
\text { Regular } \\
\text { Bueno } \\
\text { Excelente }\end{array}$ & $\begin{array}{c}0(0) \\
0(0) \\
3(20) \\
12(80)\end{array}$ & $\begin{array}{c}3(6) \\
2(4) \\
5(10) \\
38(79)\end{array}$ \\
\hline Satisfacción, mediana (RIC) & $90(20)$ & $95(20)$ \\
\hline Fuerza, mediana (RIC) & $18,4(5,2)$ & $18,2(9,4)$ \\
\hline Fuerza contralateral $(n=57)$, mediana $($ RIC $)$ & $18,35(8,4)$ & $18,8(6,5)$ \\
\hline $\begin{array}{l}\text { Se volvería a operar } \\
\text { Sí } \\
\text { No }\end{array}$ & $\begin{array}{c}15(100) \\
0(0)\end{array}$ & $\begin{array}{c}42(88) \\
6(12)\end{array}$ \\
\hline
\end{tabular}

RIC $=$ rango intercuartílico. 
Tabla 3. Comparación de las variables según el seguimiento de mediano a largo plazo

\begin{tabular}{|c|c|c|c|}
\hline Variable & Mediano plazo (\%) & Largo plazo (\%) & $\mathbf{p}$ \\
\hline Edad en años, mediana (RIC) & $64(18)$ & $58(10)$ & $0,0068^{*}$ \\
\hline $\begin{array}{l}\text { Sexo } \\
\text { Masculino } \\
\text { Femenino }\end{array}$ & $\begin{array}{c}7(21) \\
27(79)\end{array}$ & $\begin{array}{l}10(15) \\
56(85)\end{array}$ & 0,49 \\
\hline $\begin{array}{l}\text { Lateralidad } \\
\text { Derecha } \\
\text { Izquierda }\end{array}$ & $\begin{array}{l}21(62) \\
13(38)\end{array}$ & $\begin{array}{l}41(62) \\
25(38)\end{array}$ & 0,972 \\
\hline $\begin{array}{l}\text { Clasificación } \\
\text { Moderado } \\
\text { Severo }\end{array}$ & $\begin{array}{c}6(18) \\
16(47)\end{array}$ & $\begin{array}{c}9(14) \\
32(49)\end{array}$ & 0,86 \\
\hline $\begin{array}{l}\text { Anestesia } \\
\text { Local } \\
\text { Bloqueo venoso } \\
\text { General }\end{array}$ & $\begin{aligned} 28 & (82) \\
3 & (9) \\
3 & (9)\end{aligned}$ & $\begin{array}{c}58(88) \\
4(6) \\
4(6)\end{array}$ & 0,59 \\
\hline Tiempo de seguimiento en meses, mediana (RIC) & $12.5(16)$ & $48(20)$ & $\mathbf{0 , 0 0 0} *$ \\
\hline Escala de Boston, mediana (RIC) & $13(8)$ & $16(11)$ & $\mathbf{0 , 0 4 5}$ \\
\hline $\begin{array}{l}\text { Clasificación en la escala de Boston } \\
\text { Malo } \\
\text { Bueno } \\
\text { Excelente }\end{array}$ & $\begin{array}{c}4(12) \\
5(15) \\
25(73)\end{array}$ & $\begin{array}{l}13(20) \\
22(33) \\
31(47)\end{array}$ & $\mathbf{0 , 0 3 8} \mathbf{8}^{*}$ \\
\hline Escala funcional, mediana (RIC) & $10(3)$ & $11(7)$ & 0,11 \\
\hline $\begin{array}{l}\text { Clasificación en la escala funcional } \\
\text { Malo } \\
\text { Regular } \\
\text { Bueno } \\
\text { Excelente }\end{array}$ & $\begin{array}{c}1(3) \\
0(0) \\
6(18) \\
27(79)\end{array}$ & $\begin{array}{c}3(5) \\
4(6) \\
9(14) \\
50(76)\end{array}$ & 0,39 \\
\hline Satisfacción, mediana (RIC) & $90(20)$ & $90(20)$ & 0,93 \\
\hline Fuerza, mediana (RIC) & $16,7(8,4)$ & $18(8,3)$ & 0,84 \\
\hline Fuerza contralateral $(\mathrm{n}=57)$, mediana $(\mathrm{RIC})$ & $16,5(6,1)$ & $19(5,1)$ & 0,23 \\
\hline $\begin{array}{l}\text { Se volvería a operar } \\
\text { Sí } \\
\text { No }\end{array}$ & $\begin{array}{c}33(97) \\
1(3)\end{array}$ & $\begin{array}{c}61(92) \\
5(8)\end{array}$ & 0,35 \\
\hline
\end{tabular}

* $\mathrm{p}<0,05$ estadísticamente significativo. RIC $=$ rango intercuartílico. 


\section{DISCUSIÓN}

En algunos estudios, se ha demostrado que las mujeres representan hasta el 69\% de la población afectada y la media de la edad ronda los 60 años,$^{24}$ datos similares a los de este estudio, en el que había más cantidad de mujeres (80\%), con una relación mujer:hombre de 1:6,5. La mayoría de los pacientes obtuvieron resultados buenos y excelentes, según el BCTQ y la FSS, esto demuestra que la cirugía abierta de liberación de túnel carpiano con la técnica descrita es una adecuada opción terapéutica. Oteo-Álvaro y cols. ${ }^{20}$ concluyen en que la escala validada en español tiene buenas propiedades psicométricas, por lo que se decidió utilizarla en este estudio, no como factor predictivo de STC, sino como indicador de mejoría de los síntomas y el estado funcional actual, esto demuestra que los resultados a mediano y largo plazo son similares. A pesar de que el tamaño de la muestra es pequeño, este estudio es una buena aproximación para conocer los resultados a mediano y largo plazo, dado que hay pocos estudios que comuniquen los resultados a más de seis meses a causa de las dificultades en los seguimientos de estos pacientes. Los resultados buenos y excelentes correspondieron tanto a la mejoría de los síntomas como al estado funcional, una tasa de satisfacción subjetiva concordante con los datos objetivos obtenidos. La mayoría de los pacientes volvería a someterse a la cirugía teniendo en cuenta la mejoría de sus síntomas.

Las escalas BCTQ y FSS son instrumentos válidos, confiables, receptivos y, además, reflejan una buena funcionalidad y la mejoría de los síntomas en el paciente con STC. Se observó que los mejores resultados tanto del BCTQ como de la FSS correspondieron a mejores desenlaces funcionales en coincidencia con los estudios publicados. ${ }^{25,26}$ Asimismo, se observó que los pacientes con mejores puntajes en el BCTQ presentaron un mayor agarre y mejores puntajes de fuerza. Los procedimientos con resultados buenos estuvieron dentro del promedio y eran mejores que aquellos con resultados insuficientes. Por otro lado, la FSS evidenció también estar relacionada a mayor fuerza entre los grupos con excelentes resultados respecto a los otros procedimientos con resultados buenos, regulares y malos. Al igual que con el BCTQ, los procedimientos con resultados excelentes tuvieron medidas de agarre superiores a las del promedio global. Sin embargo, no se observaron diferencias en el agarre entre los procedimientos con resultados buenos, regulares y malos según la FSS.

Se obtuvieron resultados deficientes según el BCTQ categorizados como "malos" y, según la FSS, como "malos" y "regulares", en el 17\% y el 8\%, respectivamente (Tabla 2). A pesar de tener resultados deficientes según las escalas, todos los pacientes refirieron algún grado de mejoría con el procedimiento. Incluso aquellos con resultados deficientes, en su mayoría, volverían a operarse. Según el BCTQ, el 17\% tuvo procedimientos con resultados deficientes, que pueden estar relacionados con los informes de la electromiografía con diagnóstico severo; sin embargo, no se logró tener acceso al resultado de la electromiografía de todos los pacientes, por lo que no se descarta que se relacione con la presencia de comorbilidades, mayor edad, lateralidad o ganancia secundaria; no obstante, cinco de estos 17 procedimientos tuvieron una medición de fuerza por encima de la mediana; así mismo, de los ocho procedimientos con resultados regulares y malos, tres pacientes tuvieron una fuerza medida por encima de la mediana.

Los puntajes de satisfacción concuerdan con los publicados por Weber y cols., en 2010, quienes informan una tasa de satisfacción del $85 \%$ en el seguimiento a cinco años de pacientes sometidos a una descompresión del túnel carpiano, ${ }^{20}$ con una mediana del $90 \%$ tanto a mediano como a largo plazo. Todos los pacientes tuvieron algún grado de satisfacción luego de la cirugía, esto quedó reflejado en que solo el 6\% no volvería a operarse.

Este estudio, por su carácter retrospectivo, tiene limitaciones, como la pérdida de seguimiento relativamente alta: 78 pacientes que fueron excluidos del estudio por no contar con el BCTQ, la FSS, el puntaje de satisfacción global ni con la prueba de agarre con un dinamómetro; esto disminuyó el tamaño de la muestra a 100 procedimientos. Asimismo, no hay una clasificación completa en la gravedad de todos los pacientes, si bien se cuenta con datos de la electromiografía que contribuyen parcialmente como una evaluación preoperatoria, no se realizó en todos los casos y, en este estudio, solo 63 pacientes la tenían, a pesar de que se estratifica el STC en moderado y severo según la electromiografía, no hay mediciones objetivas de sensibilidad que ratificarían cada categoría de la enfermedad.

Se considera que el manejo quirúrgico del STC es una opción que logra resultados buenos y excelentes a mediano y largo plazo en cuanto a funcionalidad, fuerza y satisfacción en pacientes con STC severo o que no responde al manejo médico.

Conflicto de intereses: Los autores no declaran conflictos de intereses. 
ORCID de R. A. Hernández Ramírez: https://orcid.org/0000-0001-8467-2450 ORCID de S. E. Muñoz Medina: https://orcid.org/0000-0002-8038-298X

\section{BIBLIOGRAFÍA}

1. Chammas M. Carpal tunnel syndrome. Chir Main 2014;33(2):75-94. https://doi.org/10.1016/j.main.2013.11.010

2. Genova A, Dix O, Saefan A, Thakur M, Hassan A. Carpal tunnel syndrome: a review of literature. Cureus 2020;12(3):e7333. https://doi.org/10.7759/cureus.7333

3. Thomsen JF, Gerr F, Atroshi I. Carpal tunnel syndrome and the use of computer mouse and keyboard: a systematic review. BMC Musculoskelet Disord 2008;9:134. https://doi.org/10.1186/1471-2474-9-134

4. Lozano-Calderón S, Anthony S, Ring D. The quality and strength of evidence for etiology: example of carpal tunnel syndrome. J Hand Surg Am 2008;33(4):525-38. https://doi.org/10.1016/j.jhsa.2008.01.004

5. Palumbo CF, Szabo RM. Examination of patients for carpal tunnel syndrome sensibility, provocative, and motor testing. Hand Clin 2002;18(2):269-77. https://doi.org/10.1016/S0749-0712(01)00007-5

6. Keith MW, Masear V, Chung K, Maupin K, Andary M, Amadio PC, et al. Diagnosis of carpal tunnel syndrome. $J$ Am Acad Orthop Surg 2009;17(6):389-96. https://doi:10.5435/00124635-200906000-00007

7. Durkan JA. A new diagnostic test for carpal tunnel syndrome. J Bone Joint Surg Am 1991;73(4):535-8. Erratum in: J Bone Joint Surg Am 1992;74(2):311. PMID: 1796937

8. Chammas M, Boretto J, Burmann LM, Ramos RM, Dos Santos Neto FC, Silva JB. Carpal tunnel syndrome - Part I (anatomy, physiology, etiology and diagnosis). Rev Bras Ortop 2014;49(5):429-36. https://doi.org/10.1016/j.rboe.2014.08.001

9. Sonoo M, Menkes DL, Bland JDP, Burke D. Nerve conduction studies and EMG in carpal tunnel syndrome: Do they add value? Clin Neurophysiol Pract 2018;3:78-88. https://doi.org/10.1016/j.cnp.2018.02.005

10. Chammas M, Boretto J, Burmann LM, Ramos RM, Neto FS, Silva JB. Carpal tunnel syndrome - Part II (treatment). Rev Bras Ortop 2014;49(5):437-45. https://doi.org/10.1016/j.rboe.2014.08.002

11. Atroshi I, Hofer M, Larsson GU, Ranstam J. Extended follow-up of a randomized clinical trial of open vs endoscopic release surgery for carpal tunnel syndrome. JAMA 2015;314(13):1399-401. https://doi.org/10.1001/jama.2015.12208

12. Vasiliadis HS, Georgoulas P, Shrier I, Salanti G, Scholten RJ. Endoscopic release for carpal tunnel syndrome. Cochrane Database Syst Rev 2014;1:CD008265. https://doi.org/10.1002/14651858.CD008265.pub2

13. Sayegh ET, Strauch RJ. Open versus endoscopic carpal tunnel release: a meta-analysis of randomized controlled trials. Clin Orthop Relat Res 2015;473(3):1120-32. https://doi.org/10.1007/s11999-014-3835-z

14. Michelotti B, Romanowsky D, Hauck RM. Prospective, randomized evaluation of endoscopic versus open carpal tunnel release in bilateral carpal tunnel syndrome: an interim analysis. Ann Plast Surg 2014;73(2):S157-S160. https://doi.org/10.1097/SAP.0000000000000203

15. Domínguez A, Mira LL, Sallent A, Seijas R, Escalona C, Cugat R, et al. Short-term outcomes after median nerve release for carpal tunnel syndrome. Intern J Orthop 2017;4(3):758-62. https://doi.org/10.17554/j.issn.2311-5106.2017.04.214

16. Ishida K, Matsumoto T, Tsumura N, Kubo S, Kitagawa A, Chin T, et al. Mid-term outcomes of computer-assisted total knee arthroplasty. Knee Surg Sports Traumatol Arthrosc 2011;19(7):1107-12. https://doi.org/10.1007/s00167-010-1361-4

17. Louie D, Earp B, Blazar P. Long-term outcomes of carpal tunnel release: a critical review of the literature. Hand $(N$ Y) 2012;7(3):242-6. https://doi.org/10.1007/s11552-012-9429-х

18. Kouyoumdjian JA, Morita MPA, Molina AFP, Zanetta DMT, Sato AK, Rocha CED, et al. Long-term outcomes of symptomatic electrodiagnosed carpal tunnel syndrome. Arq Neuro Psiquiatr 2003;61(2A):194-8. https://doi.org/10.1590/S0004-282X2003000200007

19. Tang CQY, Lai SWH, Tay SC. Long-term outcome of carpal tunnel release surgery in patients with severe carpal tunnel syndrome. Bone Joint J 2017;99-B(10):1348-53. https://doi.org/10.1302/0301-620X.99B10.BJJ-2016-0587.R2

20. Oteo-Álvaro Á, Marín MT, Matas JA, Vaquero J. Validación al castellano de la escala Boston Carpal Tunnel Questionnaire. Med Clin (Barc) 2016;146(6):247-53. https://doi.org/10.1016/j.medcli.2015.10.013 
21. Andani Cervera J, Balbastre Tejedor M, Gómez Pajares F, Garrido Lahiguera R, López Ferreres A. Valoración del cuestionario de BOSTON como screening en patología laboral por síndrome del tunel carpiano. Rev Asoc Esp Espec Med Trab 2017;26:31-8. Disponible en:

http://scielo.isciii.es/scielo.php?script=sci_arttext\&pid=S1132-62552017000100004\&lng=es\&nrm=iso

22. Levine DW, Simmons BP, Koris MJ, Daltroy LH, Hohl GG, Fossel AH, et al. A self-administered questionnaire for the assessment of severity of symptoms and functional status in carpal tunnel syndrome. J Bone Joint Surg Am 1993;75(11):1585-92. https://doi.org/10.2106/00004623-199311000-00002

23. Luchetti R. Palmar incision. En: Luchetti R, Amadio P (eds.). Carpal tunnel syndrome. Berlin: Springer; 2007: 121-9.

24. Weber RA, DeSalvo DJ, Rude MJ. Five-year follow-up of carpal tunnel release in patients over age 65. J Hand Surg Am 2010;35(2):207-11. https://doi.org/10.1016/j.jhsa.2009.10.020

25. Badalamente M, Coffelt L, Elfar J, Gaston G, Hammert W, Huang J, et al. Measurement scales in clinical research of the upper extremity, part 2: outcome measures in studies of the hand/wrist and shoulder/elbow. J Hand Surg Am 2013;38(2):407-12. https://doi.org/10.1016/j.jhsa.2012.11.029

26. Leite JC, Jerosch-Herold C, Song F. A systematic review of the psychometric properties of the Boston Carpal Tunnel Questionnaire. BMC Musculoskelet Disord 2006;7:78. https://doi.org/10.1186/1471-2474-7-78 